Abstract ID: 4

\title{
The potential of Tamarindus indica seed extract as anti-snake venom against Ophiophagus hannah, Naja kaouthia and Daboia russelli in mice
}

\author{
Soraya Ismail | Pakeer Oothuman Syed Ahamed | Khin Maung Maung \\ Kulliyyah of Medicine, International Islamic University Malaysia
}

Introduction: Globally, snakebite cases are estimated to be around 5 million annually affecting mainly the poorer countries like Africa and Asia, and in 2009 WHO has categorized it as a 'neglected tropical disease'. Currently the standard treatment for snake envenomation is the anti-snake venom therapy, which is expensive, poor supply and species specific. This study was conducted to evaluate the potential of using Tamarindus indica seed extract (TSE) to inhibit the in-vitro and in-vivo effects of snake venom of three snakes; namely Ophiophagus hannah, Naja kaouthia and Daboia russelli. Methods: Methanolic extract of TSE was used. In-vitro enzymatic inhibition study i.e phospholipase $A_{2}$, proteolytic and phosphomonoesterase were studied. In-vivo acute subcutaneous (SC) toxicity of TSE in ICR mice was conducted. Study on the SC TSE inhibition of lethality was also conducted. Results: Findings from enzymatic inhibition studies showed that, TSE was able to significantly reduce $(p<0.05)$ all three enzymatic activities. SDS-PAGE experiment showed disruption of protein bands when venom reacted with TSE. No signs of toxicity were observed over 4 weeks when mice were exposed to SC TSE $60 \mathrm{mg} / 20 \mathrm{~g}$ except for superficial skin ulcers. Histological examination of the liver, both kidneys and skin showed no changes when compared to the control group. TSE was able to increase the mice survival rate. Conclusions: The study shows that SC TSE was safe to be injected up to $60 \mathrm{mg} / 20 \mathrm{~g}$ and has the potential to delay the effects of venoms from $O$. hannah, $N$. kaouthia and $D$. russelli.

KEYWORDS: Tamarind seed, anti-snake venom, plant extract, tannin, phospholipase A2 